\title{
Don't laugh at Aristotle
}

\section{There has been an amusing demonstration that heavy objects may fall to the ground faster than lighter objects, but there is not yet much of a case for resuscitating Aristotle's physics.}

THE late Leon Rosenfeld, mostly a mildmannered man, used to come nearest to rage on the subject of Aristotle, whom he would accuse of having single-handedly held back science for the best part of 2,000 years. Aristotle's physics, once turned into general orthodoxy, was especially malevolent because it was at once ingenious and intuitive. What more clever than the notion that bodies are sustained in motion by the force of the air rushing in to fill the space they vacate? And what more natural than to suppose that massive objects will fall more quickly towards the Earth than those that happen to be lighter?

Now, of course, all that has changed. Everybody knows that the pre-galileans were misled by the buoyancy and friction of the atmosphere, but - in case there should be surviving doubts - there are still a number of elementary schools at which teachers put a coin and a feather in a glass tube, evacuate the air and demonstrate that feathers can also drop like stones. That is why two physicists from the University of Massachusetts at Amherst, John F. Donoghue and Barry R. Holstein, are now entitled to a modest joke by giving a recent paper the title "Aristotle was right: heavier objects fall faster" (Eur. J. Phys. 8, 105; 1987). The effect, it will be appreciated, is only small.

In reality, this is not the first recent occasion on which a revision of the galilean opinion might have been called for. The possibility of a short-range force between massive objects dependent on the specific baryon number of the materials concerned, suggested last year by a reexamination of data collected by Eötvös in the early $1920 \mathrm{~s}$, would have made the acceleration of objects towards the Earth dependent on chemical composition. (Apparently, it remains to be seen whether that conclusion is confirmed.)

Donoghue and Holstein do not pretend to have found a new phenomenon, but merely to have calculated the consequences of the interaction between objects and the surrounding vacuum at temperatures other than absolute zero. Their account in the European Journal of Physics raises a number of intriguing issues.

The notion that the mass of an object may be a function of its temperature, and of that of the surroundings, is one of the oddest in spite of having been canvassed in the 1930 s by $R$. C. Tolman in his book
Relativity, Thermodynamics and Cosmology but on the basis of largely formal arguments in thermodynamics. The explanation now given has the virtue of making explicit a mechanism by which such an effect might arise.

But which mass? The starting-point is inevitably the formal distinction between inertial mass (the quantity in Newton's second law of motion) and gravitational mass (which appears in Newton's law of gravitation). Newton was the first to appreciate that the two masses must be identical, which also follows from the principle of equivalence, the doctrine that the laws of physics should appear the same in frames of reference moving uniformly relative to each other, the essence of which was appreciated long before the special theory of relativity. As it emerges from what Donoghue and Holstein say, it is the inertial mass that varies with temperature.

Brooding about the nature of the electron mass towards the end of the nineteenth century remains a pointer to the contemporary argument, at least in the sense of H.A.Lorentz's attempt to identify some part of the mass of a point charge with the energy of its constituent matter in its own electrostatic field; the result, numerically infinity, was a harbinger of the difficulties field theorists now resolve (or rationalize) by their technique called renormalization.

In the modern version of this argument, the vacuum is capable of supporting radiation states (materialized as photons), each of which may in principle be excited at any time, subject only to the amount of energy and momentum that is available. Indeed, at temperatures other than absolute zero, the vacuum will be filled with black-body radiation of precisely the kind defined by Planck. But, even at absolute zero, the proper calculation of the state of an isolated charge in a box requires the consideration of all possible states of the system in which photons arise spontaneously and then, to ensure conservation of energy and momentum, disappear again, again spontaneously (whence the name "virtual").

Donoghue and Holstein have several things to say, not the least interesting of which is that the expression for the energy of the system known in dynamics as the hamiltonian represents what is called the free energy in thermodynamics, and is to be identified with the inertial mass of the object, that the gravitational mass is derived from what is called the energymomentum tensor in general relativity and that the difference between the two energies (or masses) is, in thermodynamics, the product of the temperature and entropy. This boils down to the assertion that the difference between gravitational and inertial mass will be the product of temperature and the rate of change of inertial mass with temperature (other variables being constant).

By good fortune, the simplified calculations show that, even though the presence of the vacuum influences both masses at absolute zero, the effects are identical so that the principle of equivalence remains valid. Although this is not necessarily a relativistic problem, it turns out to take account of relativistic virtual states, those in which photons create fleeting electron pairs, for example.

At temperatures other than absolute zero, this simple cancellation of effects does not apply. Indeed, given that the inertial and gravitational masses differ by quantities related only to the dependence of inertial mass on temperature, it is inevitable that the two quantities should differ, and by a quantity proportional to the square of the temperature (in degrees $\mathrm{K}$ ). The gravitational mass is always less than the inertial mass. The gravitational acceleration of a charged particle near the surface of the Earth would differ from that at absolute zero by a fraction proportional to $(T / M)^{2}$, where $T$ is temperature and $M$ mass. This is the origin of Aristotle's rehabilitation; the greater the mass of an object, the less its gravitational acceleration appears to be diminished.

That at least is what the authors say. But the effect is very small; for an electron at $300 \mathrm{~K}$, the fractional departure of gravitational acceleration from that expected at absolute zero comes out at 3 parts in $10^{17}$ - some five orders of magnitude beyond what is measurable. For nucleons, whose masses are greater, the effect will be smaller, as will be that for real matter. No doubt the authors would say that the coupling of nuclear matter to other than the electromagnetic field, potentially capable of yielding similar effects, would have even smaller consequences.

The upshot, then, is not so much a vindication of Aristotle's position, but what might be called a dismissive demonstration that he was wrong from the start. John Maddox 